\title{
The Cystobactamids 920-1 and 920-2: Assignment of the Constitution and Relative Configuration by Total Synthesis
}

\author{
Therese Planke ${ }^{a \star}$, María Moreno ${ }^{a \star}$, Stephan Hüttel ${ }^{b}$, Jörg Fohrer ${ }^{\mathrm{a}}$, Franziska Gille ${ }^{\mathrm{a}}$, Matthew D. Norris ${ }^{\mathrm{a}}$, \\ Maik Siebke ${ }^{\mathrm{a}}$, Liangliang Wang ${ }^{\mathrm{a}}$, Rolf Müller ${ }^{\mathrm{b}}$, and Andreas Kirschning ${ }^{\mathrm{a} *}$ \\ a Institut für Organische Chemie und Biomolekulares Wirkstoffzentrum (BMWZ) der Leibniz Universität Hannover, Schneiderberg 1B,
30167 Hannover, Germany \\ b Abteilung Mikrobielle Naturstoffe, Helmholtz Institut für Pharmazeutische Forschung Saarland, Helmholtz Zentrum für Infektionsfor- \\ schung und Universität des Saarlandes, Campus E8.1, 66123 Saarbrücken, Germany
}

Supporting Information Placeholder

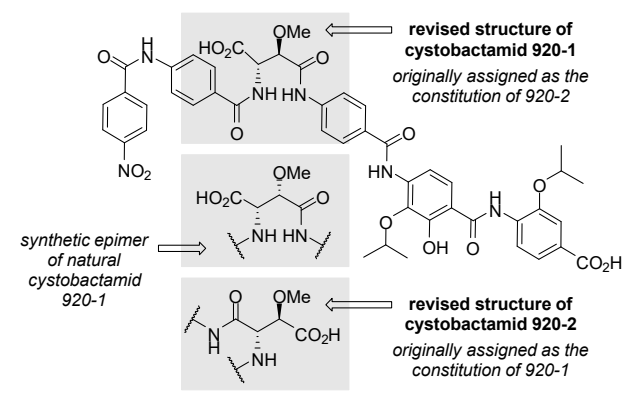

\begin{abstract}
Total synthesis of cystobactamid 920-1 and its epimer has allowed an unambiguous assignment of the relative and absolute configuration of the natural product. A careful structural analysis of each isomer using both NMR and computational techniques also prompted a constitutional revision of the structures originally reported for cystobactamids 920-1 and 920-2, and has provided further insight into the unique conformational preferences of the cystobactamid family.
\end{abstract}

In 2014, a group of nonribosomal peptides, cystobactamids 919-1 (1) and 919-2 (2), were isolated in small amounts (< $100 \mu \mathrm{g} / \mathrm{L})$ through cultivation of the myxobacterium Cystobacter sp. (Figure 1). ${ }^{1}$ Compounds $\mathbf{1}$ and $\mathbf{2}$ are closely related to albicidin, whose structure and absolute configuration was determined unequivocally by Süssmuth and coworkers through total synthesis. ${ }^{2}$ Only recently, nine new cystobactamid derivatives were isolated from Cystobacter sp., including cystobactamids 920-1 (3), 920-2 (4) and 861-2 (5); the latter being the most potent antibiotic of all known congeners. ${ }^{3}$ Indeed, the cystobactamids and albicidin show strong antibacterial activity, inhibiting several clinically relevant Gram-positive and Gram-negative strains, such as Acinetobacter baumannii ( $\mathrm{MIC}=0.5 \mu \mathrm{g} / \mathrm{mL}$ for 5), Citrobacter freundii $(\mathrm{MIC}=$ $0.06 \mu \mathrm{g} / \mathrm{mL}$ for 5), carbapenem-resistant E. coli WT-III (marR $\triangle 74 \mathrm{bp})(\mathrm{MIC}=0.5 \mu \mathrm{g} / \mathrm{mL}$ for 5), carbapenem-resistant $P$. aeruginosa (CRE) $(\mathrm{MIC}=1.0 \mu \mathrm{g} / \mathrm{mL}$ for 5) and Proteus vulgaris $(\mathrm{MIC}=0.25 \mu \mathrm{g} / \mathrm{mL}$ for 5$) .{ }^{3}$ It was also found that the cystobactamids inhibit bacterial type Ila topoisomerases, which are known to be targeted by the clinically established group of quinolone antibiotics.

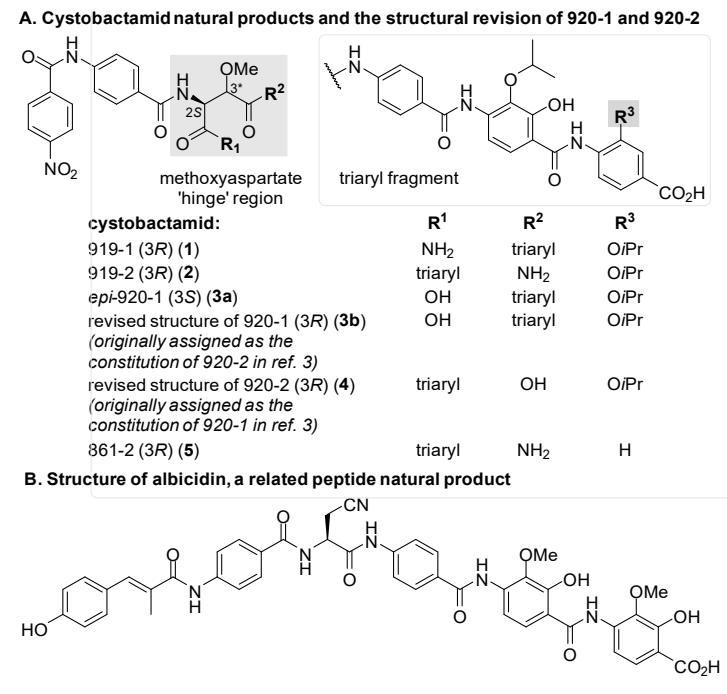

Figure 1. The cystobactamid natural products, synthetic epimer 3a and the structural revision of cystobactamids 920-1 and 920-2 as presented in this work (the related peptide albicidin is also shown). 
Total syntheses of the cystobactamids 861-2 (5) and 919-2 (4) were accomplished independently through our earlier efforts ${ }^{3}$ and that of the Trauner group, ${ }^{4}$ respectively. However, correct assignment of the two stereogenic centers in the central methoxyaspartate 'hinge' region has been a matter of debate since the original isolation paper. ${ }^{1}$ The relative configuration of $\mathbf{1}$ and $\mathbf{2}$ was originally interpreted as anti (relative to the orientation drawn in Figure 1; either $2 S, 3 S$ or $2 R, 3 R$ configuration) through comparing the homonuclear vicinal coupling constants $\left({ }^{3} J_{\mathrm{H}, \mathrm{H}}=7-8 \mathrm{~Hz}\right)$ and observed ROESY-NMR data to that of other $\beta$-oxyasparagine derivatives reported in literature. The absolute configuration of each stereocenter was similarly inferred through direct comparison of the measured optical rotation of $\mathbf{1}$ and $\mathbf{2}$ with literature compounds. Consequently, the configuration at $\mathrm{C} 2$ and $\mathrm{C} 3$ was assigned as $2 S, 3 S{ }^{1}$

Later, Kim and coworkers also isolated cystobactamid 919-2 (2) along with two other derivatives, which they named coralmycins A and B, from cultures of Corallococcus coralloides myxobacteria. ${ }^{5}$ In contrast to the original stereochemical assignment, Kim proposed that the configuration of the methoxyaspartate hinge region of $\mathbf{2}$ should be revised to $2 S, 3 R$. This alternative structural assessment also resulted from detailed NMR analysis of the natural compound, especially concerning the values of ${ }^{3} \mathrm{~J}_{\mathrm{HH}}$ and ${ }^{n} \mathrm{~J}_{\mathrm{CH}}$. Kim argued that the ${ }^{\mathrm{n}} \mathrm{J}_{\mathrm{C}, 2-\mathrm{H}}$ value of $6.7 \mathrm{~Hz}$ (in DMSO- $\mathrm{d}_{6} / \mathrm{CD}_{3} \mathrm{OD}=4: 1$ ) is consistent with a gauche conformation of 2- $\mathrm{H}$ and the methoxy group at $\mathrm{C} 3$. Additionally, it was contested that 2- $\mathrm{H}$ and $\mathrm{C} 4$, as well as $3-\mathrm{H}$ and $\mathrm{C} 1$, are gauche orientated; implying that the methoxyaspartate hinge is fixed with syn configuration (relative to the orientation drawn in Figure 1; either $2 S, 3 R$ or $2 R, 3 S$ configuration). Despite these differences in interpretation, it is notable that in both accounts the chemical shift values and coupling constant ${ }^{3} J_{2-\mathrm{H}, 3-\mathrm{H}}$ of compound $\mathbf{2}$ were identical.

In view of this ongoing stereochemical uncertainty, we endeavored to synthesize both possible epimers of the reported structure of cystobactamid 920-2, 3a and $\mathbf{3 b}$; and to perform a detailed conformational analysis of each isomer, using NMR techniques and computer-aided molecular modeling, to unambiguously assign the relative and absolute configuration of the natural cystobactamid framework. To date, neither the synthesis of a carboxylic acid bearing cystobactamid, nor that of any epimeric cystobactamid pair, have been reported.

Following our previous work on the synthesis of cystobactamid 861-2 (5) ${ }^{3,6}$ we sought to construct both epimers of the reported structure of cystobactamid 920-2 using a similar approach. Thus, the synthesis of both methoxyaspartate epimers, 10 and 12, began with methyl cinnamate (6) (Scheme 1). Similar to our earlier strategy, we envisaged that the phenyl ring in $\mathbf{6}$ would ultimately act as an effective masked carboxyl group, which may be unveiled at a later stage through chemoselective Kuhn-Roth oxidation. ${ }^{7}$

For synthesis of the $2 S, 3 S$-epimer $\mathbf{1 0}, \alpha$-acetoxy- $\beta$-bromo methylester $\boldsymbol{\gamma}$ was first prepared from $\mathbf{6}$ following a known procedure, ${ }^{8}$ which began with Sharpless asymmetric dihydroxylation as the key step (Scheme 1, part A). Nucleophilic substitution of the benzylic bromide with $\mathrm{NaN}_{3}$ yielded ester $\mathbf{8}$ with inversion of configuration, and saponification followed by $t$-butyl ester formation delivered azide $\mathbf{9}$. Notably, the latter transformation was sensitive to azido elimination under acidic conditions, necessitating use of the milder reagent $\mathrm{Me}_{2} \mathrm{~N}$ $\mathrm{CH}(\mathrm{OtBu})_{2}$. Under optimised conditions, we were pleased that this ester switch proceeded in $87 \%$ yield over two steps. With t-butyl ester $\mathbf{9}$ in hand, selective oxidation of the pendent phenyl ring to a carboxylic acid with $\mathrm{RuCl}_{3}$ and $\mathrm{NaIO}_{4}$ allowed us to simultaneously introduce the second carboxyl group of the methoxyaspartate linker, and to maintain its differentiation from the existing carboxyl moiety. This rarely employed transformation proceeded with remarkable chemoselectivity and yielded amine 10 after $O$-methylation and Staudinger reduction of the azido group.

Scheme 1. Preparation of the two epimeric methoxyaspartates.

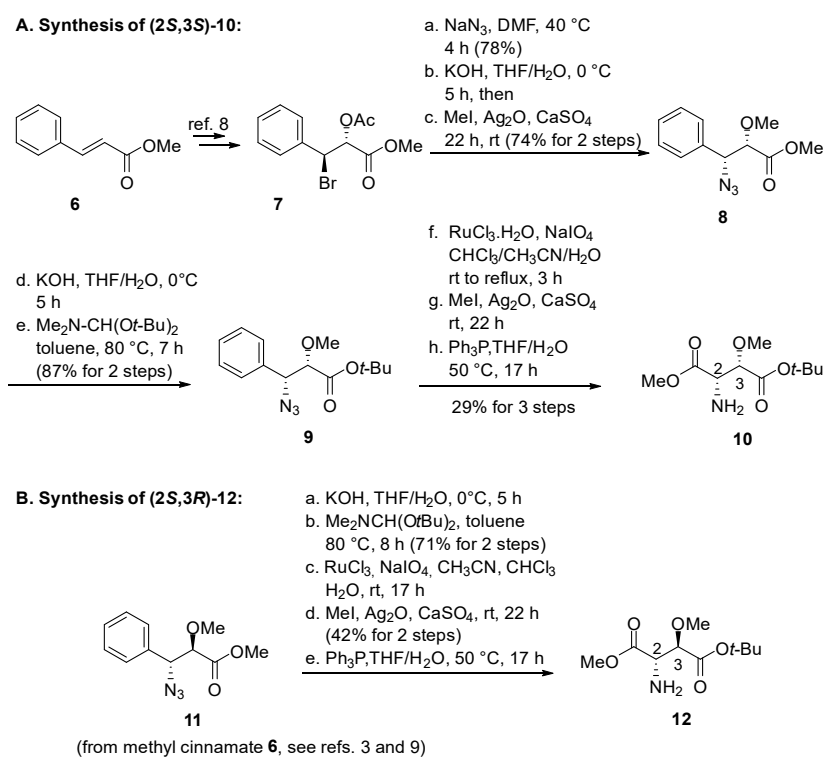

Synthesis of the 2S,3R-diastereomer $\mathbf{1 2}$ was accomplished through extension of our existing route to azide 11 from methyl cinnamate $(\boldsymbol{6}))^{3,9}$ Saponification and $t$-butyl ester formation paved the way for Kuhn-Roth oxidation, and the resulting carboxylate was advanced to amine 12 through $O$ methylation and Staudinger reduction (Scheme 1, part B).

With both epimers in hand, we proceeded to couple the eastern and western polyaromatic fragments in a stepwise approach. Best results for the first coupling reaction were realized when acid $\mathbf{1 3}^{10}$ was activated with ethyl chlorocarbonate, then added to freshly-prepared methoxyaspartate amines $\mathbf{1 0}$ or 12 in DMF (Scheme 2 and 3). Under these conditions, amides $\mathbf{1 4}$ and $\mathbf{1 7}$ were accessed in good yield from amines 10 and 12 , respectively, after $t$-butyl ester elimination. Compound 14 was then coupled to the known polyaromatic aniline fragment $\mathbf{1 5}^{3,6}$ after activation with $\mathrm{PPh}_{3} \mathrm{Cl}_{2}$ to give amide $\mathbf{1 6}$. However, under these same conditions, we were surprised to find that the epimeric acid $\mathbf{1 7}$ reacted exclusively at the phenol oxygen of 15. In order to circumvent this issue, we had to employ the known allyl protected aniline fragment $\mathbf{1 8}^{3,6}$ instead. Thus, activation of $\mathbf{1 7}$ with $\mathrm{POCl}_{3}$ in the presence of DIPEA and subsequent addition of $\mathbf{1 8}$ afforded the desired amide $\mathbf{1 9}$ in $59 \%$ yield.

Subsequent hydrolysis of the aspartate methyl ester proved to be challenging. Under conditions typical for base hydrolysis, the amino acid linker was highly sensitive to epimerization, resulting in a mixture of isomers that were difficult to separate on a preparative scale. We found that the exceptionally mild reagent $\mathrm{Me}_{3} \mathrm{SnOH}$ was best suited to affect methyl ester hydrolysis, although partial epimerization could not be 
completely suppressed. After some optimization, methyl ester 16 was successfully hydrolyzed with $\mathrm{Me}_{3} \mathrm{SnOH}$ in 1,2dichloroethane at $80{ }^{\circ} \mathrm{C}$, and $t$-butyl ester elimination afforded the $2 S, 3 S$-isomer of the originally assigned structure of cystobactamid 920-2 (3a).

Scheme 2. Preparation of $2 S, 3 S$ isomer-3a.

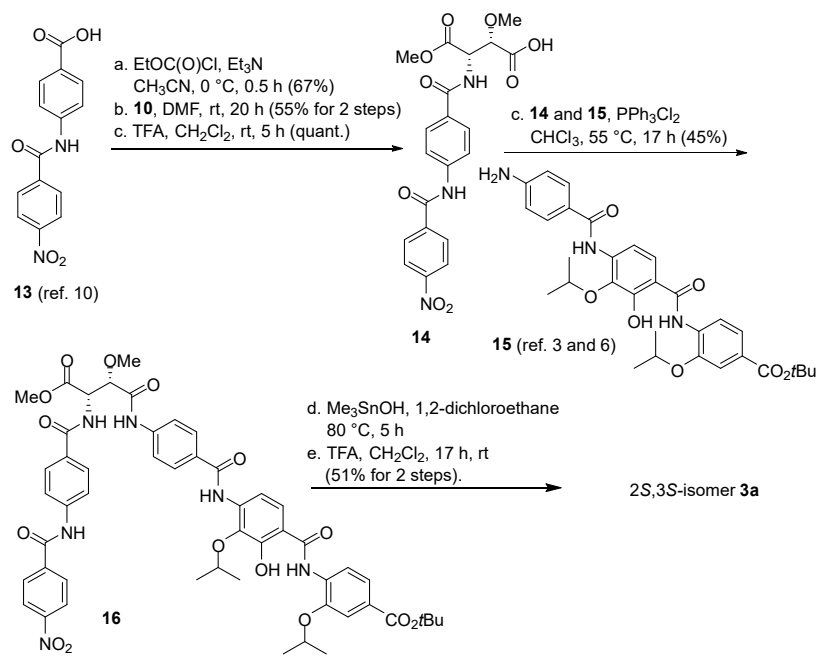

Seheme 3. Preparation of $2 S, 3 R$-isomer $\mathbf{3 b}$.
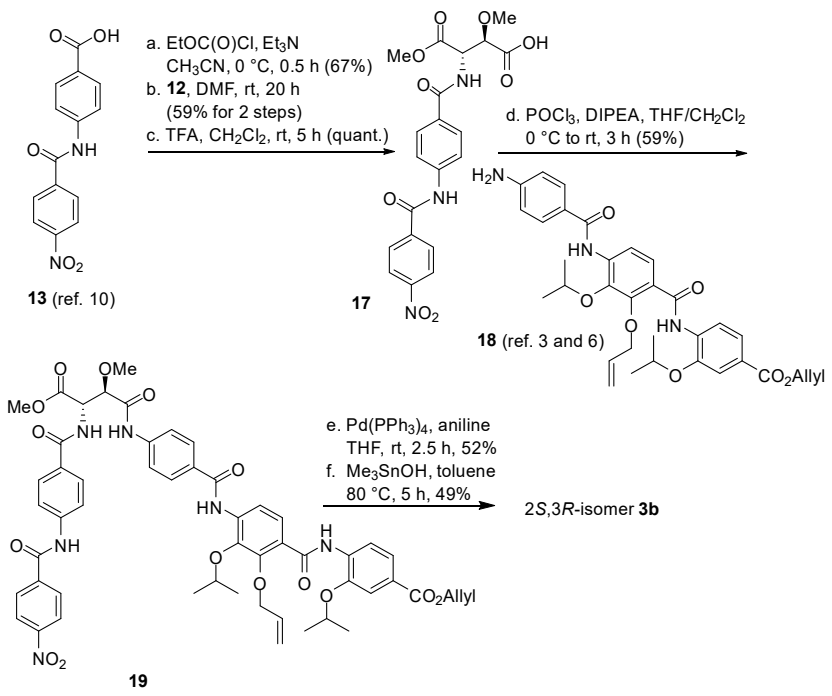

Compound $\mathbf{1 9}$ was similarly advanced to the $2 S, 3 R$-isomer of the originally assigned structure of cystobactamid 920-2 (3b), although for purification purposes, it was advantageous to perform methyl ester hydrolysis in the final step. Applying stoichiometric $\mathrm{PhSiH}_{3}$ as a scavenger in the palladium catalyzed deallylation of $\mathbf{1 9}$ was troublesome, leading to partial reduction of the aryl nitro group. Rather, aniline was found to be superior, resulting in a clean transformation to the desired product on treatment with $\mathrm{Pd}\left(\mathrm{PPh}_{3}\right)_{4}$. Curiously, hydrolysis of the remaining ester was also complicated by inadvertent alkylation of 1,2-dichloroethane (solvent) to the exposed phenol group under our previously optimized conditions; necessitating the use of toluene as solvent in this reaction. Owing to its poor solubility, the final product $\mathbf{3 b}$ was isolated in pure form using a combination of different chromatographic techniques (see Supporting Information).
Comparison of the ${ }^{1} \mathrm{H}$ NMR spectra of natural cystobactamids 920-1 and 920-2 with synthetic 3a revealed significant differences (Figure 2, part A). This included the chemical shift values $\left(\delta\right.$; designated with ${ }^{*}$ for natural $920-1$ and $*$ for natural 920-2) of one $\mathrm{N}-\mathrm{H}$ amide proton, $8.34\left(\mathrm{~d}, J=8.9 \mathrm{~Hz}, 1 \mathrm{H}_{-\mathrm{NH}}\right)$ compared to $8.72^{\#}$ (d, $J=8.67 \mathrm{~Hz}, 1 \mathrm{H}_{-\mathrm{NH}}$ ) and $8.47^{*}$ (d, $J=8.1$ $\mathrm{Hz}, 1 \mathrm{H}_{-\mathrm{NH}}$ ), and one of the $\mathrm{C}-\mathrm{H}$ protons in the methoxyaspartate hinge region, $4.48\left(\mathrm{~d}, J=4.6 \mathrm{~Hz}, 1 \mathrm{H}_{-\mathrm{CH}}\right)$ compared to $4.13^{\#}\left(\mathrm{~d}, J=8.05 \mathrm{~Hz}, 1 \mathrm{H}_{-\mathrm{CH}}\right)$ and $4.31^{*}\left(\mathrm{~d}, J=5.0 \mathrm{~Hz}, 1 \mathrm{H}_{-\mathrm{CH}}\right)$ (see atoms marked in grey in Fig. 2). From this we ultimately concluded that synthetic $\mathbf{3 a}$ is a diastereomer of natural cystobactamid 920-1. This was confirmed when comparing the ${ }^{1} \mathrm{H}-$ NMR spectra of synthetic $\mathbf{3 b}$ with natural samples of cystobactamids 920-1 and 920-2 (Figure 2, part B). Again, the amide proton and $\mathrm{CHOMe}$ group in the methoxyaspartate hinge region were diagnostic elements. Clearly, isomer $\mathbf{3 b}$ matches the authentic cystobactamid 920-1 and not its constitutional isomer cystobactamid 920-2, as it was originally proposed. Our side-by-side comparison confirms that: a) the relative configuration of the methoxyaspartate unit in both cystobactamids $920-1$ and $920-2$ is $2 S, 3 R$; and b) the constitutional assignment of each natural product must be reassigned to the structures depicted in Figure 1.
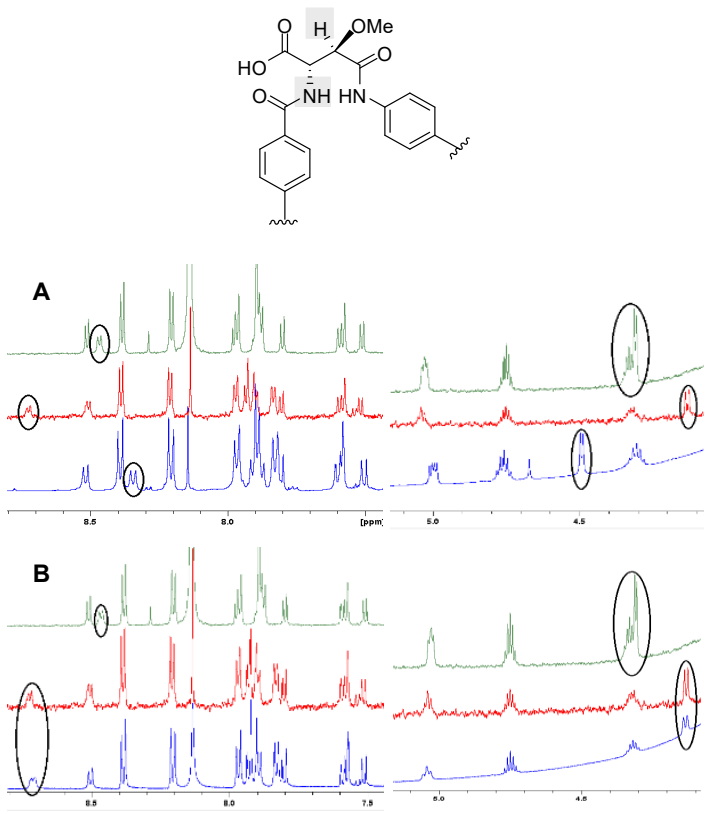

Figure 2. Comparison of the ${ }^{1} \mathrm{H}-\mathrm{NMR}$ regions of: A) synthetic 3a (blue) and B) synthetic 3b (blue), with natural cystobactamids 920-1 (red) and 920-2 (green). Left region: NH proton, right region: CHOMe proton (marked grey in the above structure).

To better understand the difficulty of structural elucidation in this family of compounds, we explored a conformational analysis of cystobactamid 920-1 (3) using the Schrödinger suite $^{12}$ maestro 9.8 computational model with the mixed torsional/low-mode sampling conformational search (LMCS) method and force field OPLS2005. ${ }^{13}$ Water was chosen as solvent and energy potentials were optimized for simulation in solution (OPLS2005) to improve the quality of force field parameters. ${ }^{14,15}$ We expected an out-of-plane orientation of the aromatic rings of $\mathbf{3}$ that might also accommodate a helical conformation. Indeed, our calculations showed that the aromatic rings of cystobactamid 920-1 (3) are not likely to preferentially be oriented in-plane. The calculations also revealed 
that the helical shape is stabilized through intramolecular hydrogen bonds between an $\mathrm{NH}$ and the phenolic group of the adjacent chain and another $\mathrm{NH}$ group of ring e with the isopropoxy group of ring b (Figure 3 , and numbering see figure 1).

Calculating the ten lowest energy conformers of cystobactamid 920-1 (3), we discovered, that the terminal aminoarene unit of the eastern fragment has greater conformational flexibility compared to the benzene rings of the western part. This observation is consistent with the work of Wilson and coworkers, who found similarly enhanced conformational flexibility in the terminal rings of oligobenzamides. ${ }^{16}$ Furthermore, the western peptide unit of cystobactamid 920-1 rotates backward to form a loop around the hinge region, such that both aromatic hemispheres are stacked on top of each other. This loop appears to be stabilized by intramolecular hydrogen bonds and hydrophobic interactions between the two chains (Figure 3). It was also possible to observe distant through-space interactions of the two polyaromatic segments using ROE spectroscopy. It is clear from the ROE spectrum of the natural compound that the amide proton at $9.58 \mathrm{ppm}$ interacts with phenyl protons of the ring vis-à-vis that resonate at 7.89 ppm as shown in Figure 3c. It must be noted that for geometrical reasons a ROE between this amide proton and the protons of the adjacent aromatic is not possible.
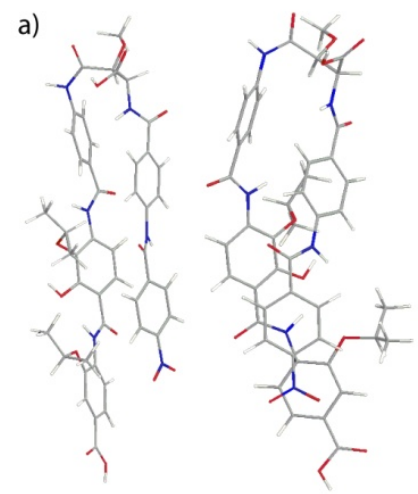

b)
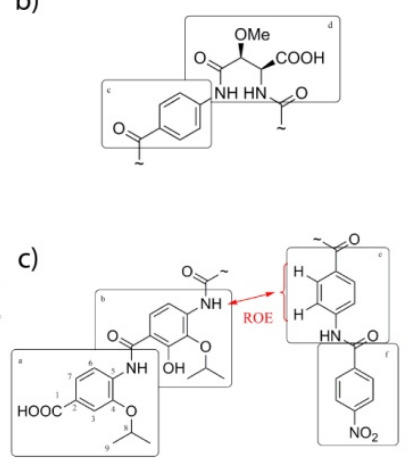

Figure 3. a) Lowest energy conformation (lowest energy conformer on the left and higher energy conformer on the right) of cystobactamid 920-1 (3) in the initially published $2 S$, $3 S$ configuration. The calculations show that both aromatic chains stack on top of each other, forming a loop around the hinge region. This loop is observed in calculations for all possible combinations of absolute configuration of the two stereocenters. b) Magnification of the hinge region with the two stereogenic centers and c) observed ROE of the natural compounds.

In summary, total synthesis of cystobactamid 920-1 (3a) and its epimer (3/b) allowed an unambiguous assignment of the relative and absolute configuration of the central methoxyaspartate unit of cystobactamid 920-1, and prompted revision of the original constitutional assignment of cystobactamids 920-1 and 920-2. A careful structural analysis using both NMR and computational techniques provided insight into the unique helical backflip conformation of the cystobactamids, which exhibit hydrogen bond interactions between the two polyaromatic arms. This strong out-of-plane preference results in an altered conformation of the methoxyaspartate hinge region, and has previously led to misconception in the structural assessment of this family of natural products.

\section{ASSOCIATEI CONTENT}

\section{Supporting Information}

The Supporting Information is available free of charge on the ACS Publications website.

Detailed experimental procedures and spectral data (PDF).

\section{AUTHOR INFORMATION}

\section{Corresponding Author}

*E-mail: andreas.kirschning@oci.uni-hannover.de.

\section{Author Contributions}

* These authors contributed equally to the work presented. Notes

The authors declare no competing financial interest.

\section{ACKNOWLEDGMENT}

This work was supported by the German Center for Infection Research (DZIF) and the Alexander-von Humboldt foundation (scholarship for M. D. Norris). We thank A. Kanakis (Leibniz Universität Hannover) for helpful support in computational calculations.

\section{REFERENCES}

(1) Baumann, S.; Herrmann, J.; Raju, R.; Steinmetz, H.; Mohr, K. I.; Hüttel, S.; Harmrolfs, K.; Stadler, M.; Müller, R. Angew. Chem. Int. Ed. 2014, 53, 14605.

(2) (a) von Eckardstein, L.; Petras, D.; Dang, T.; Cociancich, S.; Sabri, S.; Grätz, S.; Kerwat, D.; Seidel, M.; Pesic, A.; Dorrestein, P. C.; Royer, M.; Weston, J. B.; Süssmuth, R. D. Chem. Eur. J. 2017, 23, 15316. (b) Kerwat, D.; Grätz, S.; Kretz, J.; von Eckardstein, L.; Seidel, M.; Kunert, M.; Weston, J. B.; Süssmuth, R. D. Chem Med Chem 2016, 11, 1899. (c) Grätz, S.; Kerwat, D.; Kretz, J.; von Eckardstein, L.; Semsary, S.; Seidel, M.; Kunert, M.; Weston, J. B.; Süssmuth, R. D. Chem Med Chem 2016, 11, 1499. (d) Petras, D.; Kerwat, D.; Pesic,A.; Hempel, B.; von Eckardstein, L.; Semsary, S.; Arasté, J.; Marguerettaz, M.; Royer, M.; Cociancich, S.; Süssmuth, R. D. ACS Chem. Biol. 2016, 11, 1198. (e) Vieweg, L.; Kretz, J.; Pesic, A.; Kerwat, D.; Graetz, S.; Royer, M.; Cociancich, S.; Mainz, A.; Süssmuth, R. D. J. Am. Chem. Soc. 2015, 137, 7608. (f) Kretz, J.; Kerwat, D.; Schubert, V.; Grätz, S.; Pesic, A.; Semsary, S.; Cociancich, S.; Royer, M.; Süssmuth, R. D. Angew. Chem. 2015, 127, 1992. (g) Pieretti I.; Royer M.; Cociancich S.; Pesic A.; Petras D.; Süssmuth R. D. Front. in Plant Sci. 201 5, 6, 289. (h) Cociancich, S.; Pesic, D.; Petras, D.; Uhlmann, S.; Kretz, J.; Schubert, V.; Vieweg, L.; Duplan, S.; Marguerettaz, S.; Noell, J.; Pieretti, I.; Hügelland, M.; Kemper, S.; Mainz, A.; Rott, P.; Royer, M.; Süssmuth, R. D. Nat. Chem. Biol. 2015, 11, 195.

(3) Hüttel, S.; Testolin, G.; Herrmann, J.; Planke, T.; Gille, F.; Moreno, M.; Stadler, M.; Brönstrup, M.; Kirschning, A.; Müller, R. Angew. Chem. Int. Ed. 201 7, 56, 12760.

(4) Cheng, B.; Müller, R.; Trauner, D. Angew. Chem. Int. Ed. 2017, 56, 12755

(5) Kim, Y. J.; Kim, H. J.; Kim, G. W.; Cho, K.; Takahashi, S.; Koshino, H.; Kim, W. G. J. Nat. Prod. 2016, 79, 2223.

(6) Moreno, M; Elgaher, W. A. M.; Herrmann, J.; Schläger, N.; Hamed, M. M.; Baumann, S.; Müller, R.; Hartmann, R. W.; Kirschning, A. Synlett 201 5, 26, 1175

(7) Mander, L. N.; Williams, C. M. Tetrahedron 2003, 59, 1105.

(8) (a) Deng, J.; Hamada, Y.; Shiori, T. Synthesis 1998, 627. (b) Deng, J.; Hamada, Y.; Shiori, T. J. Am. Chem. Soc. 1995, 117, 7824.

(9) Sayyed, I.; Sudalai, A. Tetrahedron: Asymmetry 2004, 15, 3111.

(10) Abbel, R.; Frey, H.; Schollmeyer, D.; Kilbinger, A. Chem. Eur. J. 2005, 11, 2170.

(11) Nicolaou, K. C.; Estrada, A. A.; Zak, M.; Lee, S. H.; Safina, B. S. Angew. Chem. Int. Ed. 2005, 44, 1378. 
(12) MacroModel, version 9.8, Schrödinger, LLC: New York, NY, 2011.

(13) (a) Banks, J. L; Beard, H. S.; Cao, Y.; Cho, A.; Damm, W.; Farid. R.; Felts, A. K.; Halgren, T. A.; Mainz, D. T.; Maple, J. R.; Murphy, R.; Philipp, D. M.; Repasky, M. P.; Zhang, L. Y.; Berne, B. J.; Friesner, R. A.; Gallicchio, E.; Levy, R. M. J. Comp. Chem. 2005, 26, 1752. (b) Jorgensen, W. L.; Tirado-Rives, J. J. Am. Chem. Soc. 1988, 110, 1657.

(14) (a) DuBay, K. H.; Hall, M. L.; Hughes, T. F.; Wu, C.; Reichman, D. R.; Friesner, R. A. J. Chem. Theory Comput. 2012, 8, 4556. (b) Daniele, T.; Riccardo, Z.; Andrea, G.; Carlo, B. Chirality 2012,
24, 741. (c) C. A. Stortz, C. A.; Johnson, G. P.; French, A. D.; Csonka, G. I. Carbohydr. Res. 2009, 344, 2217.

(15) This force field is ideally suited for the conformational analysis of peptides. See: (a) Hendricks N. G.; Julian, R. R. Chem. Commun. 2015, 51, 12720. (b) Matthes, D.; de Groot, B. L. Biophys. J. 2009, 97, 599.

(16) (a) Plante, J. P; Burnley, T.; Malkova, B.; Webb, M. E.; Warriner, S. L.; Edwards, T. A.; Wilson, A. J. Chem. Commun. 2009, 5091. (b) Fuller, J. C.; Jackson, R. M.; Edwards, T. A.; Wilson, A. J.; Shirts, M. A. PLOS ONE, 2012, 7, e43253. (c) Plante, J.; Campbell, F.; Malkova B.; Kilner, C.; Warriner, S. L.; Wilson, A. J. Org. Biomol. Chem. 2008, 1, 138. 